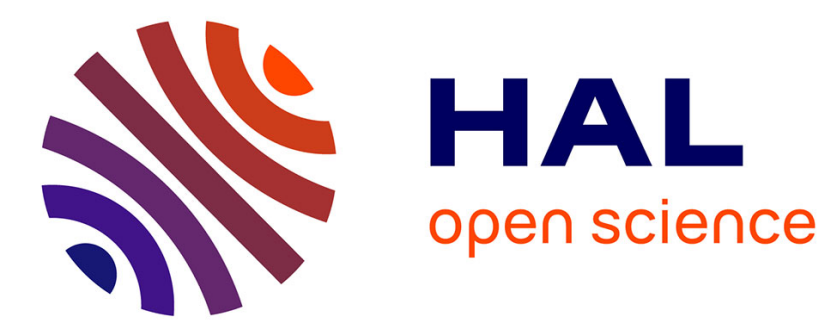

\title{
Single-Multi-Single Mode Structure Based Band Pass/Stop Fiber Optic Filter With Tunable Bandwidth
}

Saurabh Mani Tripathi, Arun Kumar, Emmanuel Marin, Jean-Pierre Meunier

\section{To cite this version:}

Saurabh Mani Tripathi, Arun Kumar, Emmanuel Marin, Jean-Pierre Meunier. Single-Multi-Single Mode Structure Based Band Pass/Stop Fiber Optic Filter With Tunable Bandwidth. Journal of Lightwave Technology, 2010, 28 (24), pp.3535-3541. hal-00549431

\section{HAL Id: hal-00549431 https://hal.science/hal-00549431}

Submitted on 22 Dec 2010

HAL is a multi-disciplinary open access archive for the deposit and dissemination of scientific research documents, whether they are published or not. The documents may come from teaching and research institutions in France or abroad, or from public or private research centers.
L'archive ouverte pluridisciplinaire HAL, est destinée au dépôt et à la diffusion de documents scientifiques de niveau recherche, publiés ou non, émanant des établissements d'enseignement et de recherche français ou étrangers, des laboratoires publics ou privés. 


\title{
Single-Multi-Single Mode Structure Based Band Pass/Stop Fiber Optic Filter With Tunable Bandwidth
}

\author{
Saurabh Mani Tripathi, Member, IEEE, OSA, Arun Kumar, OSA, Emmanuel Marin, and \\ Jean-Pierre Meunier, Member, IEEE, OSA
}

\begin{abstract}
We present a simple, efficient and easy to fabricate single-multi-single mode (SMS) fiber-based tunable bandwidth optical bandpass/bandstop filter. The device exploits the transmission characteristics of an SMS structure near its critical wavelength. Using both temperature and strain tuning, we show that the device can be switched between band pass to band stop modes and that the filter bandwidth in each mode can be dynamically tuned. We present a theoretical analysis of the observed behavior and obtain excellent agreement with the experimental observations.
\end{abstract}

Index Terms-Band pass filter, modal interference, optical fiber components, wavelength filter.

\section{INTRODUCTION}

$\mathbf{O}$ PTICAL band pass/band stop filters are an essential component in telecommunication systems where a certain range of wavelengths are required to be present or absent in the optical signal. Due to their low loss, compact size, high frequency response and ease of insertion in existing optical fiber communication channels, optical fiber filters are preferred over their electronic and integrated optical counterparts. A number of bandpass filter designs based on single-multi-single mode fiber (SMS) structures have been reported in the literature [1]-[3]. A band pass filter action can, in principle, be achieved by matching spot sizes of the fundamental modes of SMF and MMF at the filter central wavelength [1]. At wavelengths near the central wavelength, most of the light will then be coupled to the fundamental mode of the MMF and hence will be coupled out, giving a transmission maximum. Far from the central wavelength, multiple modes are excited and the transmitted power will depend upon the phase difference accumulated between the propagating modes, yielding periodic maxima and minima in the transmission spectrum. The structure will thus behave as a band pass filter for wavelengths between the two transmission minima on either side of the spot size matching wavelength. In

Manuscript received March 20, 2010; revised July 09, 2010, September 09, 2010, and October 12, 2010; accepted October 23, 2010. Date of publication October 28, 2010; date of current version December 03, 2010.

S. M. Tripathi and A. Kumar are with the Physics Department, Indian Institute of Technology Delhi, New Delhi 110016, India (e-mail: tripathi.iit@gmail.com; akumar@physics.iitd.ernet.in)

E. Marin and J. P. Meunier are with the Université de Lyon, F-42023 Saint-Etienne, France, CNRS, UMR 5516, Laboratoire Hubert Curien, F-42000 Saint-Etienne, France and also with the Université de Saint-Etienne, Jean-Monnet, F-42000 Saint-Etienne, France (e-mail: emmanuel. marin@univ-st-etienne.fr; meunier@univ-st-etienne.fr).

Color versions of one or more of the figures in this paper are available online at http://ieeexplore.ieee.org.

Digital Object Identifier 10.1109/JLT.2010.2090035 such a scheme, however, the filter bandwidth will be extremely large as the spot sizes of SMF/MMF do not vary very strongly with the wavelength. In another scheme, the bandpass filter action is achieved by selecting the MMF length in such a way that, at a certain wavelength, the field distribution at its output end is the image of the input field [2], [3]. Thus, there is a peak in the transmission at this wavelength, and at other wavelengths where self-imaging does not occur, the transmitted power is reduced. The advantage of this scheme is that the central filter wavelength can be easily tuned to another value by simply changing the MMF length so that the self imaging for the new MMF length is now achieved at another wavelength. Optical fiber gratings have also been widely used to realize wavelength filters. For narrow bandwidth applications, fiber Bragg gratings (FBG) can be used for both bandstop filtering (operating in the transmission mode) and bandpass filtering (operating in the reflection mode) [4], [5]. Long period gratings can also be used as a platform to form tunable bandpass filters, for example, by using a combination of LPGs in a partially core removed fiber [6], by inducing a $\pi$-phase shift in the grating [7], or by using an acousto-optic LPG with either a core block [8], [9] or hollow fiber to remove the uncoupled light [10]. For bandstop applications several other schemes based on long period gratings (LPGs) [5], [11], [12] and liquid crystal filled photonic crystal fibers [13]-[15] have been reported. In the case of LPGs, for higher order cladding modes (typically $\mathrm{LP}_{015}$ or higher in SMF-28 ${ }^{\mathrm{TM}}$ ) at a specific (critical) wavelength ' $\lambda_{c}$ ' the cladding mode group index equals that of the core mode, leading to a turning point of the phase matching (grating period versus resonance wavelength) curve at $\lambda_{c}$ [16]. Thus, in LPGs operated near $\lambda_{c}$ of a given higher order cladding mode, there are two resonance wavelengths corresponding to the same grating period, and this fact can be exploited to generate tunable band pass/band stop filters.

Apart from the filters based on SMS structures, however, all the filters we described require complex fabrication techniques and therefore increase the cost of the system. Furthermore, the filters based on UV-written gratings can only be operated in low temperature environments, as even annealed devices will eventually degrade and bleach over time at ambient temperatures exceeding $250-300^{\circ} \mathrm{C}$. An optical filter which is simple and low cost, yet versatile in that its central wavelength and bandwidth can be tuned and it can be switched between bandpass and bandstop modes, would be definitely more attractive than the above filters.

In this paper we present a simple and easy to fabricate SMS fiber-based bandwidth-tunable optical bandpass/bandstop filter. We utilize the transmission properties of the SMS structure 


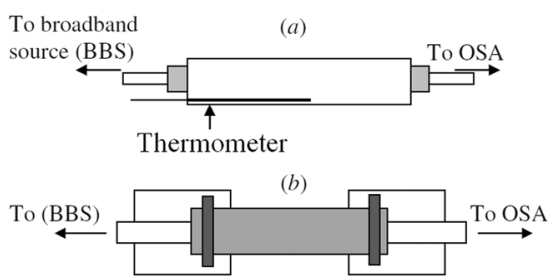

Fig. 1. Schematic diagram of the SMS structure based filter using (a) thermal variation and (b) axial strain.

around $\lambda_{\mathrm{c}}$. The proposed mechanism is basically a special case of self imaging such that the self-imaging at the output end of the MMF is achieved at the critical wavelength $\lambda_{c}$. This provides an extra degree of freedom of tuning the SMS structure to either pass or stop the transmission over a specific bandwidth. We show that the transmission/rejection bandwidth can be adjusted to a desired value by selecting the MMF length appropriately and by applying an external perturbation in the form of axial strain or temperature to the MMF section.

\section{Filter Structure AND EXPERIMENTAl Results}

A schematic diagram of the SMS structure based filter used in our experiments is shown in Figs. 1(a), (b). The sample is prepared by splicing a $40 \mathrm{~cm}$ long section of $\mathrm{GeO}_{2}$ doped MMF (iXfiber-Ge), using a Fujikura fusion splicer (FSM 40S), in between two SMFs (SMF-28 ${ }^{\mathrm{TM}}$ ). The loss at both splices was $\sim 0.02 \mathrm{~dB}$. Light is launched from a broadband source to the lead-in SMF, through the device to the lead-out SMF and spectrally resolved using an optical spectrum analyzer (OSA) (HP 70004A). At the first splice, the light is coupled to various modes of the MMF which then propagate with their respective propagation constants and accumulate a certain phase difference over the length of the MMF, which ultimately leads an interference pattern in the measured spectrum. In order to show the bandwidth tuning and the switching between band pass and band stop regimes, a small section $(l=30 \mathrm{~cm})$ of the MMF section is heated by passing it through a computer controlled furnace and the transmission spectrum is continuously recorded.

In Fig. 2(a) we plot the transmission spectrum of the SMS structure at three different temperatures, $\mathrm{T}=27^{\circ} \mathrm{C}$ (solid curve), $\mathrm{T}=32^{\circ} \mathrm{C}$ (dashed-dotted curve) and $65^{\circ} \mathrm{C}$ (dashed curve), with a wavelength resolution of $0.1 \mathrm{~nm}$. The input power spectrum is also shown, indicating an insertion loss of $\sim 1.5$ $\mathrm{dB}$ over the range of operating wavelengths. The figure clearly shows that by heating the MMF the structure can be made to behave as a band pass or band stop filter with adjustable bandwidth. For example, at $\mathrm{T}=27^{\circ} \mathrm{C}$ the structure behaves as band pass filter with a full width at half maximum (FWHM) bandwidth of $\sim 26 \mathrm{~nm}$, while at $\mathrm{T}=32^{\circ} \mathrm{C}$, the FWHM broadens to $\sim 38 \mathrm{~nm}$. The corresponding filter roll off at these temperatures is $\sim 0.33 \mathrm{~dB} / \mathrm{nm}$ and $\sim 0.5 \mathrm{~dB} / \mathrm{nm}$, respectively. At $\mathrm{T}=65^{\circ} \mathrm{C}$, the structure switches over to band stop mode with the FWHM and filter roll off of $\sim 41 \mathrm{~nm}$ and $0.3 \mathrm{~dB} / \mathrm{nm}$, respectively. Similar behavior is observed with respect to applying axial strain. This is demonstrated in our second experiment, where we employed a slightly shorter length $(1-10 \mathrm{~cm})$ of the MMF.
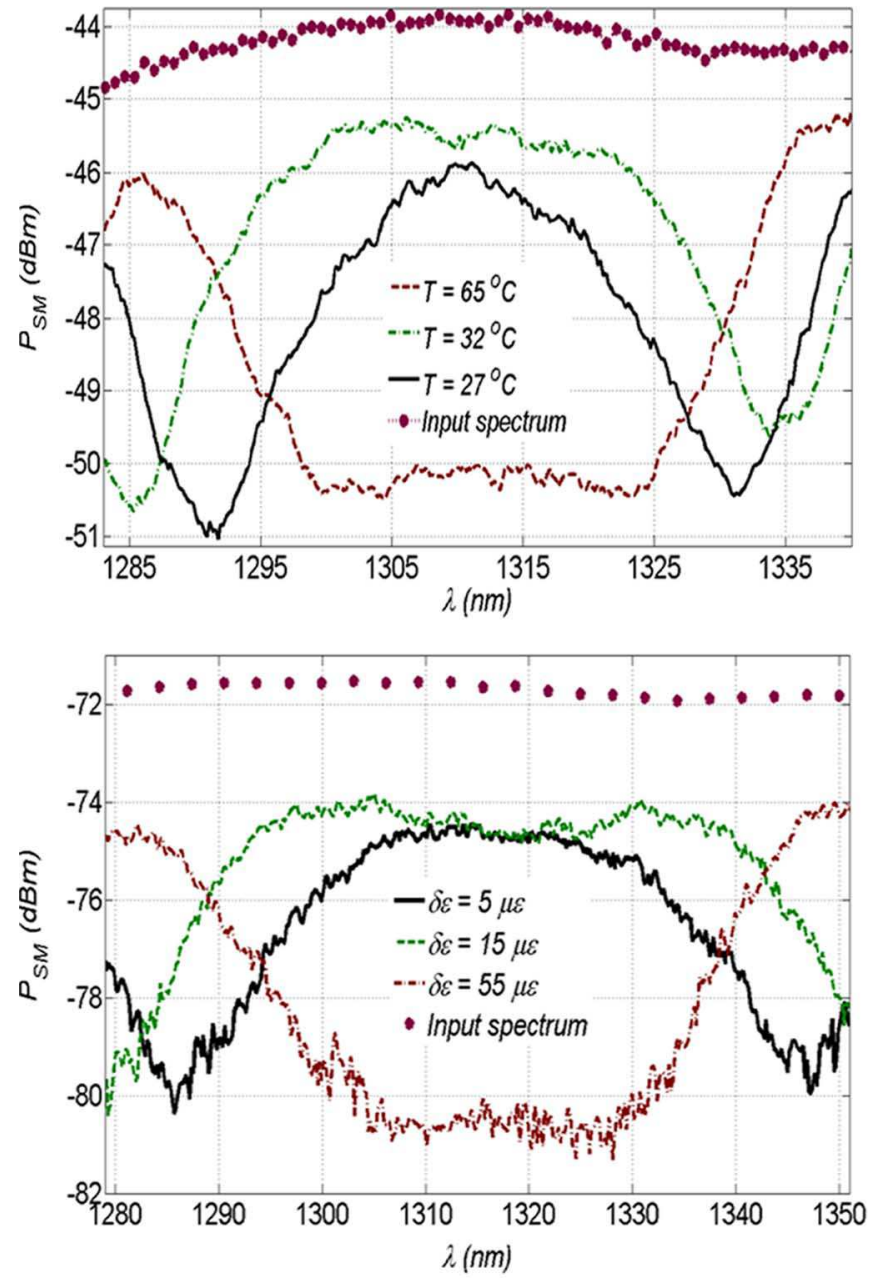

Fig. 2. Experimental transmission spectra showing the variation of transmission bandwidth in band pass regime with respect to changing (a) temperature (b) axial strain at room temperature.

In Fig. 2(b) ${ }^{1}$ we show the influence of axial strain on the transmission bandwidth and on the filter action. We again plot the input power spectrum, showing an insertion loss of $\sim 2 \mathrm{~dB}$ over the range of operating wavelengths. We note that since the experiments were performed at two different laboratories, two different samples were prepared for temperature and strain measurements. The longer MMF length used in temperature tuning experiment helps to reduce the temperature change required for switching from band pass to band stop operation as the small induced index change gets multiplied by the propagation length, whereas the shorter fiber length in the strain tuning experiment reduces the elongation necessary to achieve a given strain. In Fig. 2(b)) the transmission spectrum has been plotted at three different axial strains: $5 \mu \varepsilon$ (solid curve), 15 $\mu \varepsilon$ (dashed-dotted curve) and $55 \mu \varepsilon$ (dashed curve). In order not to disturb the launch and collection conditions of the SMS structure while applying the axial strain, the MMF section was fixed to a stationary platform near the first splice and connected to a micropositioner near the other splice. The strain was then

${ }^{1}$ The difference in the power levels in Fig. 2(a) and (b) is due to the fact that Exp.1 was carried out with a supercontinuum source at LabHC, France, whereas Exp. 2 was carried out with a broadband source HP 70951B at IIT Delhi, India. 
applied by stretching the MMF with an increment of $2 \mu \mathrm{m}$ and the transmission spectrum was continuously recorded. At $5 \mu \varepsilon$ and $15 \mu \varepsilon$, the spectrum shows a band pass response with the FWHM $\sim 44 \mathrm{~nm}$ and $\sim 57 \mathrm{~nm}$ and a corresponding filter roll off of $\sim 0.32 \mathrm{~dB} / \mathrm{nm}$ and $\sim 0.29 \mathrm{~dB} / \mathrm{nm}$, respectively. At $55 \mu \varepsilon$, the filter switches over to the band stop mode with the FWHM $\sim 46$ $\mathrm{nm}$ and filter roll off of $0.31 \mathrm{~dB} / \mathrm{nm}$. The fluctuations observed in the experimental curves in the band stop regime are due to the detector noise, since the input power level is extremely low.

In Fig. 3(a) we show the variation of FWHM as a function of temperature; the variation of the central wavelength of the filter (critical wavelength) with temperature is shown in the inset. The figure consists of both the experimental (shown by error bar) as well as theoretical (shown by solid line) results obtained by using the analysis discussed in the next section. The bandwidth varies almost linearly with temperature with a slope of $\sim 2.1 \mathrm{~nm} /{ }^{\circ} \mathrm{C}$, whereas the central wavelength varies much more slowly with the slope of $\sim 0.04 \mathrm{~nm} /{ }^{\circ} \mathrm{C}$. Similar variation of the FWHM and the central wavelength as a function of applied axial strain is shown in Fig. 3(b), where the bandwidth varies with a slope of $\sim 0.1 \mathrm{~nm} / \mu \varepsilon$ and the central wavelength varies with a slope of $\sim 0.03 \mathrm{~nm} / \mu \varepsilon$. We note that unlike fiber gratings, SMS structures based devices can be used at much higher temperatures [17]. In our experiments, for example, no significant change in the filter characteristics were observed up to $\sim 600^{\circ} \mathrm{C}$ after which the central filter wavelength starts deviating from the linear path.

\section{THEORETICAL ANALYSIS}

In this section we present a theoretical analysis of the observed filter action using a simple and accurate analysis based on the modal interference among guided modes of the MMF section. In our analysis, the fibers are considered to be axially aligned so that only the symmetric modes of the MMF are excited and the well known linear polarization (LP) approximation is used to analyze the modal propagation. This is a good approximation in the present case, as the splice loss is very small and the index contrast of the fibers is weak. Only a few low order modes of the MMF typically carry most of the power coupled to the MMF. Nonetheless, we take into account all the guided modes of the MMF in our calculations. The field at the beginning of the MMF section can be expressed as $\Psi_{M} \equiv \sum_{i} a_{i} \Psi_{i}$ where $\Psi_{i}$ is the normalized spatial field profile of the $i$ th mode of the MMF and $a_{i}$ is its corresponding field amplitude at the lead-in splice. The field amplitude $a_{i}$ is determined by the modal overlap between the fundamental mode of the SMF and the $i$ th mode of the MMF, so if we define $\Psi_{S} \equiv \Psi_{S}(r)$ as the normalized fundamental mode field of the SMF, $a_{i}$ may be written as

$$
a_{i}=2 \pi \int_{0}^{\infty} \Psi_{S} \Psi_{i}^{*} r d r
$$

Different guided modes have different propagation constants, so they will develop a phase difference while propagating along the MMF length. At the lead-out splice these fields are then coupled
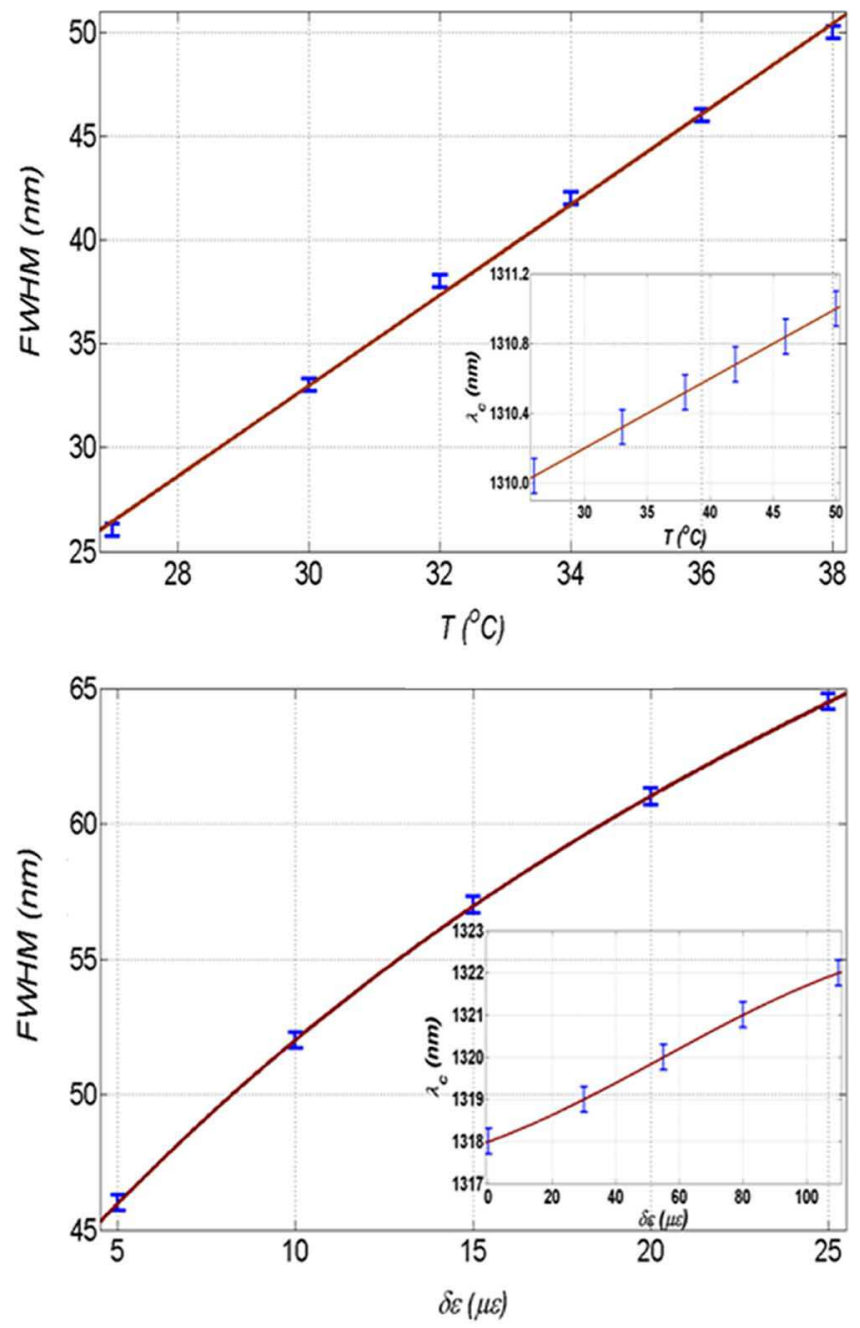

Fig. 3. Experimental (error bar) and theoretical (solid line) curves showing the variation of FWHM of the filter with (a) temperature and (b) applied strain. The variation of central wavelength $\left(\lambda_{c}\right)$ is shown in the inset.

back to fundamental mode of the SMF, and the power in the lead-out fiber can be written as [18]

$$
P_{\mathrm{SM}}=\left|a_{0}^{2}+a_{1}^{2} e^{i\left(\beta_{0}-\beta_{1}\right) L}+a_{2}^{2} e^{i\left(\beta_{0}-\beta_{2}\right) L}+\cdots\right|^{2}
$$

where, $\beta_{i}$ is the propagation constant of $i$ th mode and $L$ is the length of the MMF section.

The iXFiber-Ge MMF used in the experiments has a staircase refractive index profile containing five layers [19] where peak doping concentration of $\mathrm{GeO}_{2}$ at the fiber axis is $11 \mathrm{wt} \%$. The LP modes of the above MMF and their corresponding propagation constants for the MMF are obtained using the matrix method [20]. The wavelength dependence of the refractive indices of various layers have been taken into account by using the Sellmeier relation [21].

\section{A. Effect of Temperature}

Temperature dependence of the refractive index has been obtained by using the relation $n_{i}=n_{0 i}+\left(\left(d n_{0 i}\right) /(d T)\right)\left(T-T_{0}\right)$, where $n_{0 i}$ represents the refractive indices at room temperature $T_{0}$, and the subscript ' $i$ ' stands for cladding or core region. 
The thermo-optic coefficient $\left(d n_{0 i}\right) /(d T)$ for fused silica and $15 \mathrm{~mol} \% \mathrm{GeO}_{2}$ doped silica are $1.06 \times 10^{-5} /{ }^{\circ} \mathrm{C}$ and $1.24 \times$ $10^{-5} /{ }^{\circ} \mathrm{C}$, respectively [22]. In order to estimate the position-dependent thermo-optic coefficient of the graded index MMF core, we assume it depends linearly on Ge concentration [23]. The changes in length and core radius with temperature are taken as $\Delta l=\alpha l \Delta T$ and $\Delta a_{M}=\alpha a_{M} \Delta T$ respectively. Here $\alpha\left(=5.0 \times 10^{-7} /{ }^{\circ} \mathrm{C}\right)$ is the thermal expansion coefficient for fused silica [24].

Since only a section (length ' $l$ ') of the MMF (length ' $L$ ') is being heated the phase factor appearing in (1) must be modified as

$$
\begin{aligned}
\left|\left(\beta_{0}-\beta_{m}\right) L\right|_{T}=\left|\left(\beta_{0}-\beta_{m}\right)(L-l)\right|_{T_{0}} \\
\quad+\left|\left(\tilde{\beta}_{0}-\tilde{\beta}_{m}\right)(l+\Delta l)\right|_{T}
\end{aligned}
$$

where $\tilde{\beta}_{i}$ is temperature dependent propagation constant of the $i$ th mode. We note that the thermo-optic coefficient is two orders of magnitude larger than the thermal expansion coefficient, so changes in the glass index is the dominant physical mechanism governing the temperature tuning.

\section{B. Effect of Strain}

The changes in refractive index and the core radius due to strain are calculated using the following equations [24]:

$$
\begin{aligned}
n_{i} & =n_{0 i}+\left\{\frac{\partial n_{0 i}}{\partial l} \Delta l\right\}=n_{0 i}+\left\{\frac{n_{0 i}^{3}}{2 l}\left[p_{12}-\nu\left(p_{11}+p_{12}\right)\right] \Delta l\right\} \\
a_{M} & =a_{0 M}+\frac{\partial a_{0 M}}{\partial l} \Delta l=a_{0 M}-\frac{a_{0 M} \nu}{l} \Delta l
\end{aligned}
$$

For fused silica we have taken, $p_{11}=0.12, p_{12}=0.27$ and $\nu=0.17$. Of the three physical processes which can affect the modal phase accumulation under strain tuning, the change in the MMF length dominates, whereas the strain optic effect adds a small correction and the change in core radius can usually be neglected.

\section{THEORETICAL RESUlTS AND Discussion}

The transmission spectrum of the given SMS structure is calculated using (1)-(4). In Figs. 4(a), (b) we plot the transmission at different temperatures or axial strains. We use the experimental parameters for the fiber length in our calculations, in that we set the total MMF length to be $40 \mathrm{~cm}$, whereas the heated section is $30 \mathrm{~cm}$ and the strained section is $10 \mathrm{~cm}$. Just as in the experiments, the calculated spectrum shows an increase in the bandwidth as the temperature is increased from $27^{\circ} \mathrm{C}$ to $32^{\circ} \mathrm{C}$ (or as the strain is increased from $5 \mu \varepsilon$ to $15 \mu \varepsilon$ ), and a switch from the bandpass regime to the bandstop regime at $\mathrm{T}=65^{\circ} \mathrm{C}$ for the temperature based filter (or $\Delta l / l=55 \mu \varepsilon$ for axial strain based filter). Our modeling shows that most of the power in the MMF is carried by the $\mathrm{LP}_{01}$ and $\mathrm{LP}_{02}$ modes only. Specifically, we find that $a_{0}^{2}=67 \%$ and $a_{1}^{2}=21 \%$ at $\lambda=1.3 \mu \mathrm{m}$, or $88 \%$ of the input power is carried by these two modes of the MMF. We can thus greatly simplify the analysis by considering only two modes and write (2) as

$$
P_{\mathrm{SM}} \simeq a_{0}^{4}+a_{1}^{4}+2 a_{0}^{2} a_{1}^{2} \cos \left(\left(\beta_{0}-\beta_{1}\right) L\right) .
$$
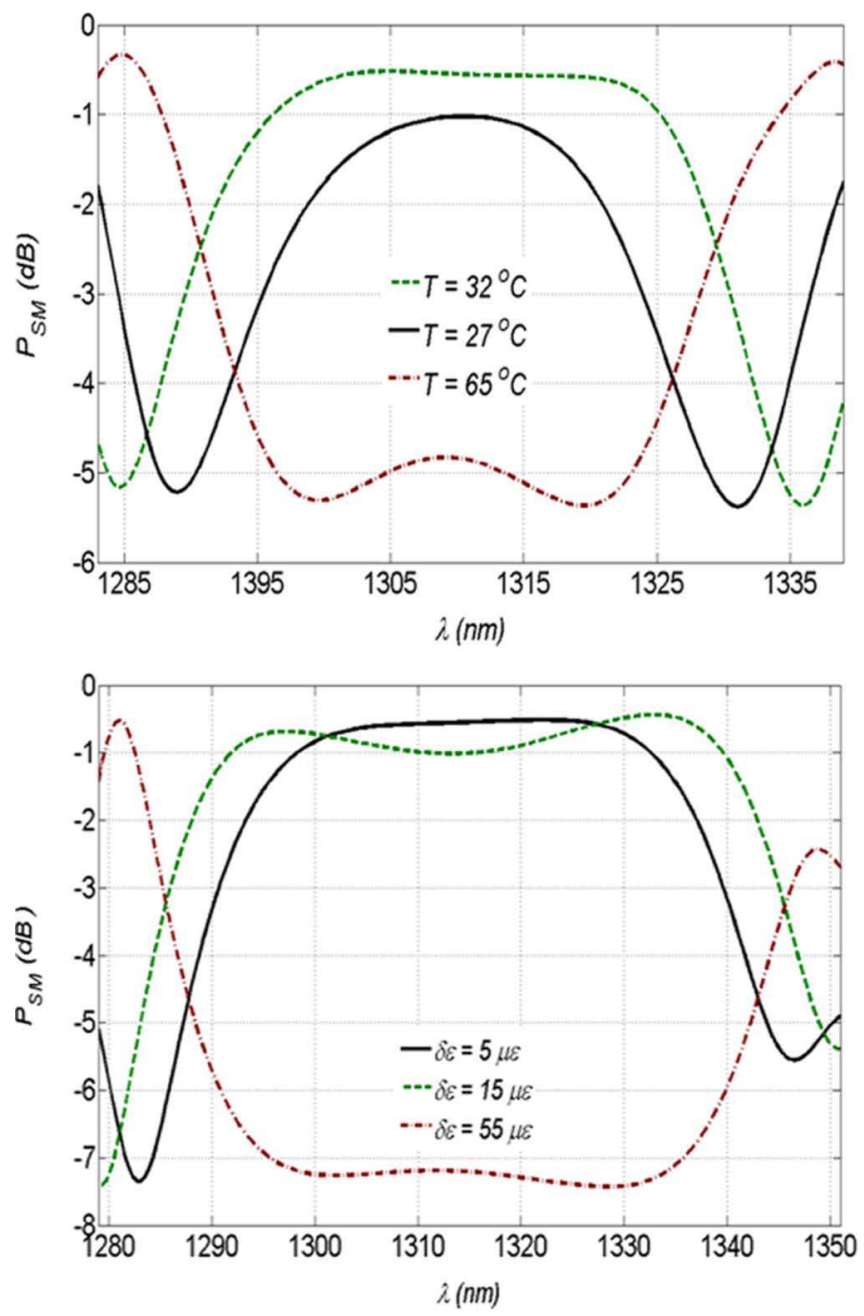

Fig. 4. Theoretical transmission spectra showing the switching from band pass regime to band stop regime with respect to changing (a) temperature (b) axial strain.

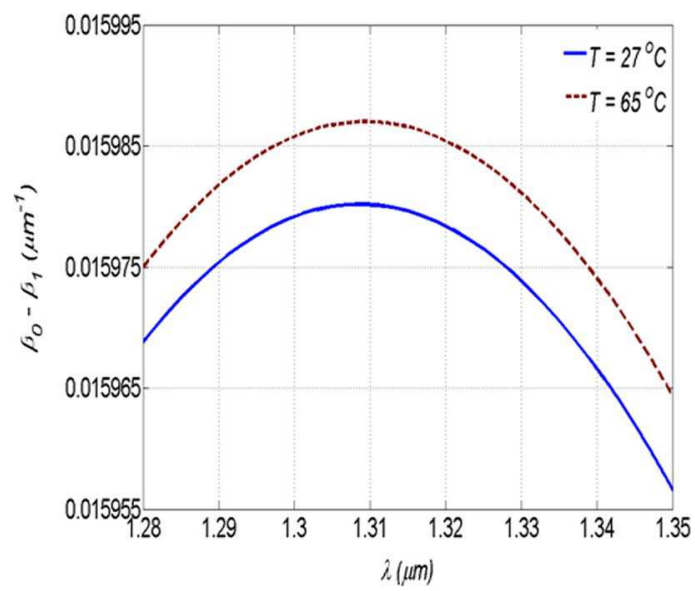

Fig. 5. Spectral variation of the propagation constant difference $\left(\beta_{0}-\beta_{1}\right)$, at $\mathrm{T}=27^{\circ} \mathrm{C}$ (solid curve) and $\mathrm{T}=65^{\circ} \mathrm{C}$ (dashed curve).

So we obtain transmission maxima at $\phi=\left(\beta_{0}-\beta_{1}\right) \mathrm{L}=2 \mathrm{n} \pi$, and transmission minima at $\phi=(2 \mathrm{n}+1) \pi, \quad n=0,1,2, \ldots$ Fig. 5 shows the variation of $\left(\beta_{0}-\beta_{1}\right)$ with wavelength at two different temperatures, where the maxima of the curves define 

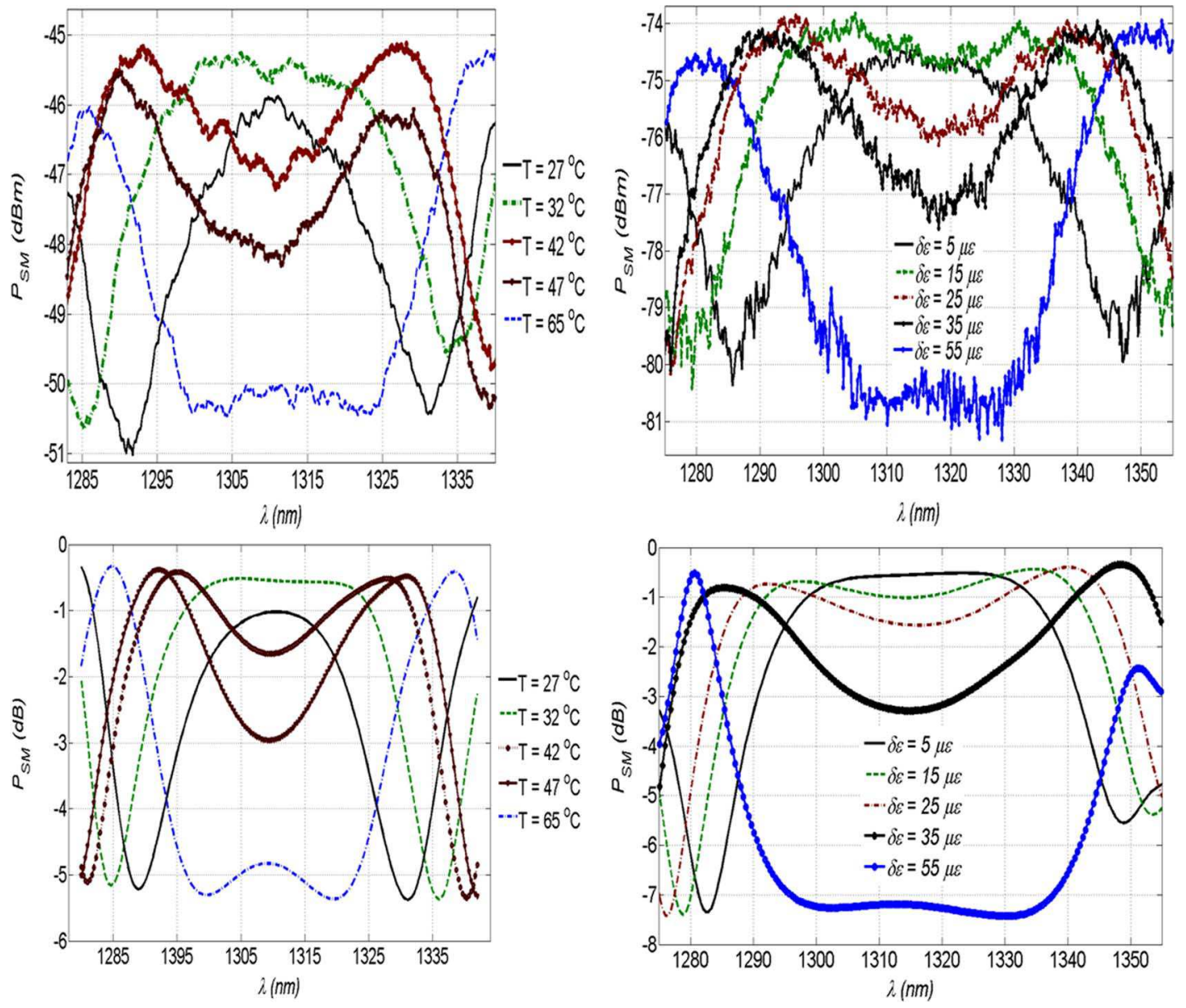

Fig. 6. Transmission spectra showing evolution from band pass regime to band stop regime with respect to change in (a) temperature (b) axial strain. Theoretical spectrum with respect to change in (c) temperature and (d) strain.

the critical wavelength $\lambda_{\mathrm{c}}$. Since $\left(\beta_{0}-\beta_{1}\right)$ varies very slowly with wavelength near $\lambda_{\mathrm{c}}$, the structure can behave as either a band pass or band stop filter with a flat response near $\lambda_{c}$. The filter response can be switched by applying sufficient heat or axial strain to the MMF to change $\phi$ by $\pi$. In Fig. 5, the values of $\left(\beta_{0}-\beta_{1}\right)$ at diffetent temperatures are obtained by calculating $\beta_{0}$ and $\beta_{1}$ using the matrix method [20] taking into account the temperature induced changes in the core and cladding refractive indices as well as in the core radius.

Tuning the filter bandwidth can be explained in a similar manner. The maximum transmission at $\lambda_{c}$ is obtained when $\phi=2 \mathrm{n} \pi$, so if we apply temperature or strain to increase the optical path length and phase accumulation in the MMF, then $\phi>2 \mathrm{n} \pi$ and $\cos (\phi)<1$ leading to a slightly reduced transmission power at $\lambda_{c}$. The propagation constant difference $\beta_{0}-\beta_{1}$ at $\lambda_{\mathrm{c}}$ is always a global maximum (Fig. 5), so as one moves on either side of $\lambda_{c}, \beta_{0}-\beta_{1}$ decreases and $\phi$ moves towards $2 n \pi$. We thus get two closely spaced transmission maxima increasing the filter bandwidth. These maxima separate from each other in wavelength as the temperature or strain is increased. We directly compare the experimentally measured and numerically simulated transmission spectra under temperature and strain tuning in Fig. 6. In all cases, as the applied perturbation is increased the two dips/peaks move apart and the transmission at $\lambda_{c}$ decreases. As the perturbation is increased further the transmission at $\lambda_{c}$ quickly approaches a minimum, thus switching from bandpass mode to bandstop mode. By increasing the perturbation even further, the transmission at $\lambda_{c}$ goes back up and the same process is repeated in reverse. In our calculated spectra, the FWHM can be tuned between $49 \mathrm{~nm}-60 \mathrm{~nm}$ with a slope of $\sim 2.2 \mathrm{~nm} /{ }^{\circ} \mathrm{C}$ or $0.11 \mathrm{~nm} / \mu \varepsilon$, and these values agree well with the corresponding experimental values of $47 \mathrm{~nm}-59 \mathrm{~nm}, 2.1 \mathrm{~nm} /{ }^{\circ} \mathrm{C}$ and $0.1 \mathrm{~nm} / \mu \varepsilon$, respectively. Discrepancies between the experimental results and the theoretical predictions can be attributed to the fact that the refractive index and thermo/strain optic coefficients of different layers used in our calculations may be slightly different than the exact values of the experimental fiber.

Using the two mode approximation, we now derive equations for the various performance characteristics so as to better understand the limiting factors which govern the device design.

1) FWHM Bandwidth: From (5) it follows that the maximum and minimum powers coupled to the lead-out SMF are $P_{\mathrm{SM}}^{\max }=$ $\left(a_{0}^{2}+a_{1}^{2}\right)^{2}$ and $P_{\mathrm{SM}}^{\min }=\left(a_{0}^{2}-a_{1}^{2}\right)^{2}$. If we assume $\phi\left(\lambda_{c}\right)=n \pi$, we may estimate the bandwidth as the difference between two wavelengths $\lambda_{1}$ and $\lambda_{2}$ on either side of $\lambda_{\mathrm{c}}$ such that $P_{\mathrm{SM}}\left(\lambda_{1}\right)=$ $P_{\mathrm{SM}}\left(\lambda_{2}\right)=\left(P_{\mathrm{SM}}^{\max }+P_{\mathrm{SM}}^{\min }\right) / 2$ giving $\phi\left(\lambda_{\mathrm{c}}\right)-\phi\left(\lambda_{1}\right)=\phi\left(\lambda_{2}\right)-$ 


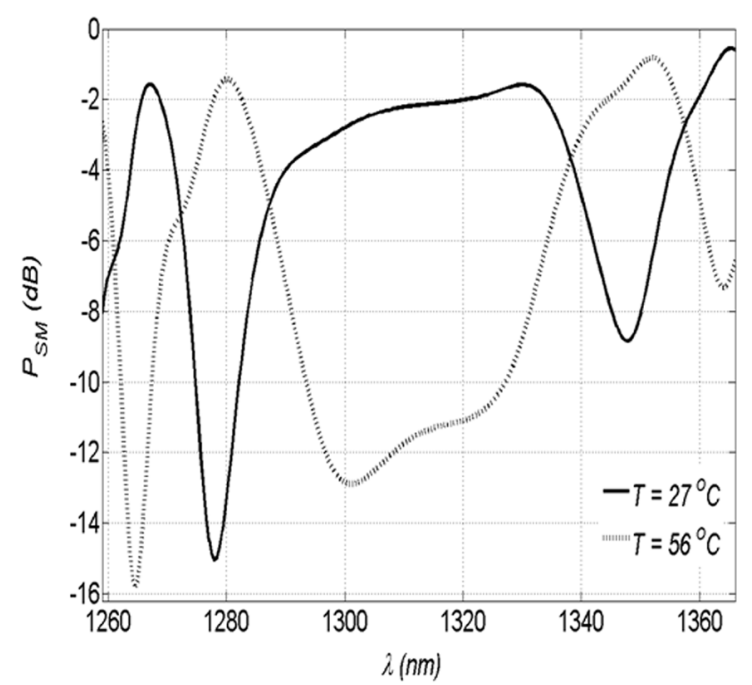

Fig. 7. Temperature induced filter action of the SMS structure employing a SMF with core diameter of $4 \mu \mathrm{m}$ doped with $4.1 \mathrm{~mol} \%$ of $\mathrm{GeO}_{2}$. The MMF parameters and its length are same as used throughout the paper.

$\phi\left(\lambda_{c}\right)=\pi / 2$. Following a Taylor series expansion of $\phi\left(\lambda_{1,2}\right)$ near $\lambda_{c}$, the bandwidth may be approximated as

$$
\mathrm{FWHM}=2 \sqrt{\frac{\pi}{L}\left(\left.\frac{d^{2}\left(\beta_{0}-\beta_{1}\right)}{d \lambda^{2}}\right|_{\lambda_{c}}\right)^{-1}} .
$$

In the above, we have used the fact that $\left(d\left(\beta_{0}-\beta_{1}\right)\right) /\left(\left.d \lambda\right|_{\lambda_{c}}=\right.$ 0 . Substituting $L=40 \mathrm{~cm}$, at $T=27^{\circ} \mathrm{C}$ the FWHM bandwidth is calculated as $\sim 25 \mathrm{~nm}$, which matches very well with the experimental value of $\sim 26 \mathrm{~nm}$. It should be noted that (6) gives the FWHM only if the phase difference at $\lambda_{c}$ is an integral multiple of $\pi$. For a general case, if $\phi\left(\lambda_{c}\right)=n \pi+\phi_{0}$, then wavelengths $\lambda_{1}$ and $\lambda_{2}$ on either side of $\lambda_{\mathrm{c}}$ will correspond to $\phi\left(\lambda_{c}\right)-\phi\left(\lambda_{1}\right)=\phi\left(\lambda_{2}\right)-\phi\left(\lambda_{c}\right)=\pi / 2+\phi_{0}$ and (6) will be modified to

$$
\mathrm{FWHM}=2 \sqrt{\frac{\left(\pi+2 \phi_{0}\right)}{L}\left(\left.\frac{d^{2}\left(\beta_{0}-\beta_{1}\right)}{d \lambda^{2}}\right|_{\lambda_{c}}\right)^{-1}} .
$$

2) Dynamic Range: The extinction ratio or the dynamic range of the filter is given by $r_{e}=10 \log \left(P_{\mathrm{SM}}^{\max }\right) /\left(P_{\mathrm{SM}}^{\min }\right)=$ $10 \log \left(\left(a_{0}^{2}+a_{1}^{2}\right) /\left(a_{0}^{2}-a_{1}^{2}\right)\right)^{2}$. Substituting $a_{0}^{2}=0.67$ and $a_{1}^{2}=0.21$, the dynamic range is calculated as $\sim 5.64 \mathrm{~dB}$, which matches very well with the experimental value. It is clear from the expression for $r_{\mathrm{e}}$ that the dynamic range can be increased by reducing the difference between $a_{0}^{2}$ and $a_{1}^{2}$, which is governed by the modal overlap between the fundamental mode of the SMF and the different modes of the MMF at the input splice. This can be achieved by selecting the SMF or MMF spotsize such that $\left(a_{0}^{2}-a_{1}^{2}\right)$ is minimum. For example, our calculations show that for the MMF used in our experiments, the extinction ratio can be increased to $10-12 \mathrm{~dB}$ by using a SMF with core diameter $\sim 4 \mu \mathrm{m}$ doped with $4.1 \mathrm{~mol} \%$ of $\mathrm{GeO}_{2}$. The corresponding transmission spectrum with thermal tuning is shown in Fig. 7. However, the increased dynamic range in this case comes at the expense of an increased insertion loss, $\sim 2 \mathrm{~dB}$ here as compared to $\sim 0.5 \mathrm{~dB}$ in Fig. 6 .

We thus present two simple guidelines for designing a tunable band pass/band stop wavelength filter utilizing the multimode interference effect near the critical wavelength.

1) The doping concentration and the refractive index profile of the MMF should be selected to obtain the central filter wavelength at the critical wavelength. The MMF length should be then selected to achieve the desired filter BW. Larger MMF lengths give smaller BWs.

2) The lead-in and lead-out SMFs should be selected such that the difference in powers coupled to first two dominant modes $\left(\mathrm{LP}_{01}\right.$ and $\left.\mathrm{LP}_{02}\right)$ of the MMF is minimum. This can be achieved by properly selecting the core diameter of the SMF.

\section{CONCLUSION}

In summary we have demonstrated a tunable bandpass/bandstop filter with tunable transmission bandwidth using multimode interference in an optical fiber. The filter action is based on utilizing the zero group index difference between the $\mathrm{LP}_{01}$ and $\mathrm{LP}_{02}$ modes of the MMF section. We show that a switch over from bandpass to bandstop regime as well as the tuning of the filter bandwidth in each regime can be achieved by introducing an appropriate change in the temperature/axial strain of the MMF.

\section{REFERENCES}

[1] A. Kumar, R. K. Varshney, S. Antony C., and P. Sharma, "Transmission characteristics of SMS fiber optic sensor structures," Opt. Commun., vol. 219, pp. 215-219, 2003.

[2] W. S. Mohammed, P. W. E. Smith, and X. Gu, "All-fiber multimode interference bandpass filter," Opt. Lett., vol. 31, pp. 2547-2549, 2006.

[3] J. E. A. Lopez, A. C. Guzman, D. A. M. Arrioja, R. S. Aguilar, and P. L. K. Wa, "Tunable multimode-interference bandpass fiber filter," Opt. Lett., vol. 35, pp. 324-326, 2010.

[4] K. O. Hill, Y. Fujii, D. C. Johnson, and B. S. Kawasaki, "Photosensitivity in optical fiber waveguides: Application to reflection filter fabrication," Appl. Phys. Lett., vol. 32, pp. 647-649, 1978.

[5] R. Kashyap, Fiber Bragg Gratings. : : Academic Press, 1999.

[6] D. S. Starodubov, V. Grubsky, and J. Feiberg, "All-fiber bandpass filter with adjustable transmission using cladding-mode coupling," IEEE Photon. Technol. Lett., vol. 10, no. 11, pp. 1590-1592, Nov. 1998.

[7] H. Ke, K. S. Chiang, and J. H. Peng, "Analysis of phase-shifted longperiod fiber gratings," IEEE Photon. Technol. Lett., vol. 10, no. 11, pp. 1596-1598, Nov. 1998.

[8] D. A. Satorius, T. E. Dimmick, and G. L. Burdge, "Double-pass acoustooptic tunable bandpass filter with zero frequency shift and reduced polarization sensitivity," IEEE Photon. Technol. Lett., vol. 14, no. 9, pp. 1324-1326, Sep. 2002.

[9] K. J. Lee, D. I. Yeom, and B. Y. Kim, "Narrow band polarization insensitive all fiber acousto-optic tunable bandpass filter," Opt. Exp., vol. 15, pp. 2987-2992, 2007.

[10] S. Choi, T. J. Eom, B. H. Lee, J. W. Lee, and K. Oh, "Broad-band tunable all-fiber bandpass filter based on hollow optical fiber and long period-grating pair," IEEE Photon. Technol. Lett., vol. 17, no. 1, pp. 115-117, Jan. 2005.

[11] A. M. Vengsarkar, P. J. Lemaire, J. B. Judkins, V. Bhatia, T. Erdogan, and J. E. Sipe, "Long-period fiber gratings as band-rejection filters," $J$. Lightw. Technol., vol. 14, no. 1, pp. 58-65, Jan. 1996.

[12] V. Dangui, M. J. Digonnet, and G. S. Kinos, "Ultrabroadband singlemode long-period fiber grating using highe-order cladding modes," $J$. Appl. Phys., vol. 96, pp. 5987-5991, 2004.

[13] T. T. Larsen and A. Bjarklev, "Optical devices based on liquid crystal photonic bandgap fibres," Opt. Exp., vol. 20, pp. 2589-2596, 2003. 
[14] L. Scolari, T. T. Akeskjold, J. Riishede, and A. Bjarklev, "Continuously tunable devices based on electrical control of dual-frequency liquid crystal filled photonic bandgap fibers," Opt. Exp., vol. 13, pp. 7483-7496, 2005.

[15] L. Scolari, S. Gauza, H. Xianyu, L. Zhai, L. Eskildsen, T. T. Akeskjold, S.-T. Wu, and A. Bjarklev, "Frequency tunability of solid-core photonic crystal fibers filled with nanoparticle-doped liquid crystals," Opt. Exp., vol. 17, pp. 3754-3764, 2009.

[16] X. Shu, X. Zhu, Q. Wang, S. Jiang, W. Shi, Z. Huang, and D. Huang, "Dual resonance peaks of $\mathrm{LP}_{015}$ cladding mode in long-period grating," Electron. Lett., vol. 35, pp. 649-650, 1999.

[17] E. B. Li, X. Wang, and C. Zhang, "Fiber-optic temperature sensor based on interference of selective higher-order modes," Appl. Phys. Lett., vol. 89, pp. 091119-3, 2006.

[18] S. M. Tripathi, A. Kumar, R. K. Varshney, Y. B. P. Kumar, E. Marin, and J.-P. Meunier, "Strain and temperature sensing characteristics of single-mode-multimode-single-mode structures," J. Lightw. Technol., vol. 27 , no. 13 , pp. 2348-2356, Jul. 2009.

[19] G. Origlio, M. Cannas, S. Girard, R. Boscaino, A. Boukenter, and Y. Ouerdane, "Influence of the drawing process on the defect generation in multistep-index germanium-doped optical fibers," Opt. Lett., vol. 34, pp. 2282-2284, 2009.

[20] C. Vassallo, Optical Waveguide Concepts. New York: Elsevier, 1991, pp. 112-114.

[21] M. J. Adams, An Introduction to Optical Waveguides. New York: Wiley, 1981

[22] Y.-J. Kim, U.-C. Paek, and B. H. Lee, "Measurement of refractiveindex variation with temperature by use of long-period fiber gratings," Opt. Lett., vol. 27, pp. 1297-1299, 2002.

[23] J. B. MacChesney, Personal Communication Aug. 2001, Bell Laboratories, Lucent Technologies, 600 Mountain Avenue, Murray Hill, N.J..

[24] S.-Y. Huang, J. N. Blake, and B. Y. Kim, "Perturbation effects on mode propagation in highly elliptical core two-mode fibers," J. Lightw. Technol., vol. 8, no. 1, pp. 23-33, Jan. 1990.

Saurabh Mani Tripathi was born in Gorakhpur, India, on May 29, 1982. He received the B.Sc. and M.Sc. degrees from Gorakhpur University, India, in 2003 and 2005, respectively, and the Ph.D. degree from the Indian Institute of Technology Delhi, New Delhi, India, in 2010.

He has been an Eiffel Excellence Fellow at the Laboratory Hubert Curien, Saint-Etienne, France, where he carried out part of his research during 2008-2009. His research interests are modal interference based guided wave devices, integrated optical devices, optical waveguide gratings and surface plasmon polariton based sensors and devices.

Dr. Tripathi is a member of the Optical Society of America and IEEE Photonics Society.
Arun Kumar received the M.Sc. and Ph.D. degrees in physics from the Indian Institute of Technology Delhi, New Delhi, India, in 1972 and 1976, respectively.

Since 1977, he has been a member of the faculty of the Physics Department, IITD, where he has been a Professor since 1995. He was a Visiting Scientist with the Technical University of Hamburg-Harburg, Germany, as a Humboldt Research Fellow in 1980-1981; the Opto-electronic Group, Strathclyde University, Glasgow, U.K., in 1988; the National Institute of Standards and Technology, Boulder, CO, in 1993 and 1994; the University of Nice, France, in 1996; and the University of Jean Monnet, Saint-Etienne, France in 1999, 2004, 2006 and 2007. He has authored or coauthored more than 95 research papers in the international journals. His research interests are in the field of optical waveguides, fiber optic sensors, and polarization mode dispersion.

Prof. Kumar is a member of the Optical Society of America.

Emmanuel Marin was born in Valence, France, on February 24, 1970. He received the Ph.D. degree from the University Jean Monnet, Saint-Etienne, France, in 2000.

His doctoral work focused on the prediction of Bragg grating effects in optical fiber components. After postdoctoral work on phononic properties of microstructured optical fibers at Bath University, Bath, U.K., he has been with the Department of Physics, University Jean Monnet, as an Assistant Professor since 2001. His current research activities include fiber-optic sensors and waveguides.

Jean-Pierre Meunier (M'01) graduated from the University of Lyon I, France, in 1971. He received the Diplôme d'Etudes Approfondies degree in quantum mechanics and the Doctorat de Spécialité degree in theoretical nuclear physics from the Nuclear Physics Institute of Lyon, France, in 1972 and 1973, respectively, and the Doctorat d'Etat ès Science degree in physics from the University Jean Monnet, Saint-Etienne, France, in 1986.

Since 1974, he has been with the department of Physics, University Jean Monnet, where he is currently a Professor working in the signal and electromagnetic theory, integrated and guided wave optics, and applied mathematics. In 1976, he cofounded a laboratory and transferred to work on optical waveguides. His current research interests are in developing theoretical tools for the modeling and characterization of passive and active optical guided wave devices. He has authored or coauthored more than 135 research papers in the international journals and conference proceedings. He is editor of two books on optical fibers for telecommunications.

Prof. Meunier is a member of the French Optical Society, the Optical Society of America, and IEEE Photonics Society. 